\title{
Diagnostic, analyse et modélisation du bruit et des chemins de bruit dans une cabine d'hélicoptère
}

\author{
Julien Caillet $^{1, a}$, François Malburet $^{1}$, Jean-Claude Carmona ${ }^{1}$ et Franck Marrot ${ }^{2}$ \\ 1 Laboratoire des Sciences de l'Information et des Systèmes, UMR CNRS 6168, CER ENSAM, 2 cours des Arts et Métiers, \\ 13617 Aix-en-Provence, France \\ 2 EUROCOPTER France, service Vibration et Bruit Interne, Aéroport international de Marseille-Provence, \\ 13725 Marignane Cedex, France
}

Reçu le 2 novembre 2004 ; accepté le 26 septembre 2006

Résumé - La démarche de cette étude s'inscrit dans le cadre de la volonté politique d'Eurocopter France
d'améliorer le confort acoustique des cabines d'hélicoptère, le but étant d'identifier les contributions des
différentes sources de bruit sur le niveau de bruit interne et d'analyser les chemins de propagation (solidiens
et aériens) de l'énergie depuis son origine jusqu'à la cabine. La gamme de fréquence analysée est celle du
dB SIL4 (353-5650 Hz), qui permet de caractériser les perturbations engendrées sur l'intelligibilité d'une
conversation. Ce travail a pour but de conduire à préconiser des solutions techniques pour améliorer le
confort acoustique des matériels et notamment des panneaux d'habillage. La modélisation de la réponse
acoustique de la cabine est réalisée à partir de mesures expérimentales réalisées en vol afin de prendre en
compte tous les phénomènes associés au déplacement et à la déformation de la structure. Dans un premier
temps, les différentes méthodes de diagnostic de bruit interne et de localisation de sources acoustiques
sont recensées dans l'optique de statuer sur le système le mieux adapté au milieu hélicoptère. Les outils de
simulation utilisés pour exploiter les résultats obtenus et répondre aux objectifs visés sont ensuite présentés.

Mots clés : Hélicoptère / localisation de sources / holographie / imagerie acoustique / formation de voies / lancer de faisceaux / acoustique géométrique

\begin{abstract}
Diagnosis, analysis and modelling of noise paths in a helicopter cabin. This study aims at improving acoustical comfort in helicopter cabins by identifying the different contributions of noise sources on internal noise level and understanding the way sound propagates through the airframe and reaches into the cabin via airborne and structure-borne energy paths, thus producing radiated noise by the panels of the cabin. The frequency band at stake here is the range of dBSIL4 (Speech Interference Level, 4-octave average). This study should lead to propose some efficient noise reduction techniques (trim panels, joints). This work will rely upon in-flight measurements in order to take into account all the deformation and vibrating phenomena, and a modelling technique will allow us to rebuild acoustic pressure at the passenger's ears location. The different diagnostic techniques are discussed in order to derive the best one according to the measurement obtained in a helicopter cabin. The simulation tools used to illustrate the results thus obtained and to reach the expected goals, are then presented.
\end{abstract}

Key words: Helicopter / source localization / nearfield acoustical holography / acoustical imaging / beamforming / geometrical acoustics

\section{Introduction}

Cette étude a pour but d'améliorer le confort acoustique des cabines d'hélicoptère. La gamme de fréquence analysée ici est celle du dB SIL4 (353-5650 Hz), qui

\footnotetext{
a Auteur correspondant :

julien.caillet@eurocopter.com
}

permet de caractériser les perturbations engendrées sur l'intelligibilité d'une conversation.

L'amélioration du confort acoustique suppose la compréhension et la maîtrise des mécanismes physiques responsables de l'ambiance sonore. En pratique, on distingue trois phénomènes essentiels : des sources de bruit dites principales, des voies solidiennes et aériennes de transmission énergétique, et des émissions sonores dues 


\section{Nomenclature}

\begin{tabular}{|ll}
\hline$\omega:$ & Pulsation $\left(\right.$ rad. $\left.\mathrm{s}^{-1}\right)$ \\
$c:$ & Célérité du son dans l'air $\left({\left.\mathrm{m} . \mathrm{s}^{-1}\right)}\right)$ \\
$k_{x}:$ & Nombre d'onde $\left(\mathrm{rad} . \mathrm{m}^{-1}\right)$ \\
$k_{y}:$ & Nombre d'onde $\left(\mathrm{rad} . \mathrm{m}^{-1}\right)$ dans la direction $x$ \\
$G:$ & Fonction de Green \\
$p_{\mathrm{S}}:$ & Champ de pression acoustique complexe dans le plan Source \\
$p_{\mathrm{A}}:$ & Champ de pression acoustique complexe dans le plan Antenne \\
$P_{\mathrm{S}}:$ & Transformée de Fourier spatiale de la pression acoustique dans le plan Source \\
$P_{\mathrm{A}}:$ & Transformée de Fourier spatiale de la pression acoustique dans le plan Antenne \\
$V:$ & Champ de vitesse normale à la surface source $\left(\mathrm{m} . \mathrm{s}^{-1}\right)$ \\
\hline
\end{tabular}

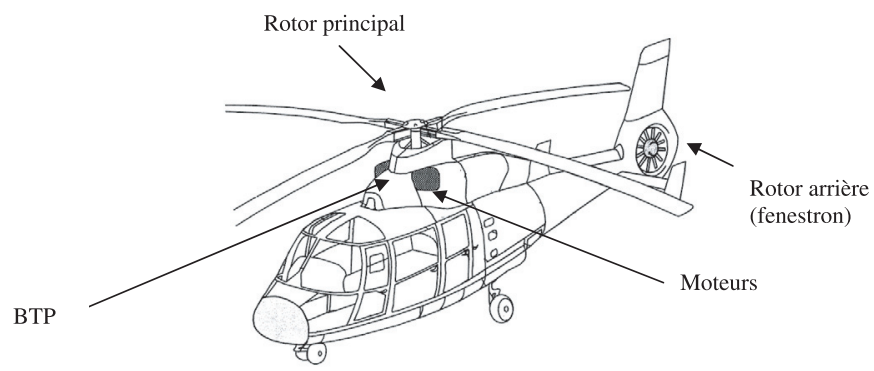

Fig. 1. Sources de bruit principales sur un hélicoptère Dauphin.

à la vibration des différents éléments de la cabine (panneaux, cadres de la structure).

La figure 1 met en évidence les sources de bruit identifiées sur les hélicoptères.

Parmi ces sources, le rotor principal produit un bruit large bande dû aux turbulences et un bruit basse fréquence $(\approx 20 \mathrm{~Hz})$ à très fort niveau $(\approx 100 \mathrm{dBlin}$ en cabine). Les moteurs sont une autre source importante qui produit un bruit large bande à cause des écoulements dans les tuyères ainsi que des sons hautes fréquences $(\approx 10 \mathrm{kHz})$. Ces deux sources restent a priori secondaires dans la gêne acoustique. La plus forte gêne semble a priori provoquée par la $\mathrm{BTP}^{1}$ qui produit un spectre de raies à très fort niveau dans la bande $1000 \mathrm{~Hz}-4000 \mathrm{~Hz}$. Cette bande correspondant au maximum de sensibilité de l'oreille est centrée sur notre bande de fréquences d'études, et c'est donc la première source à traiter pour améliorer le confort des passagers. À ces sources viennent s'ajouter le bruit aérodynamique d'avancement largebande et les bruits de chauffage et de ventilation.

En conséquence, l'allure du spectre de bruit mesuré en cabine est de la forme (Fig. 2).

\section{Les chemins de bruit}

Les bruits sont globalement transférés par voie aérienne, mais dans le cas de la BTP, le couplage avec

\footnotetext{
1 Boîte de Transmission Principale.
}

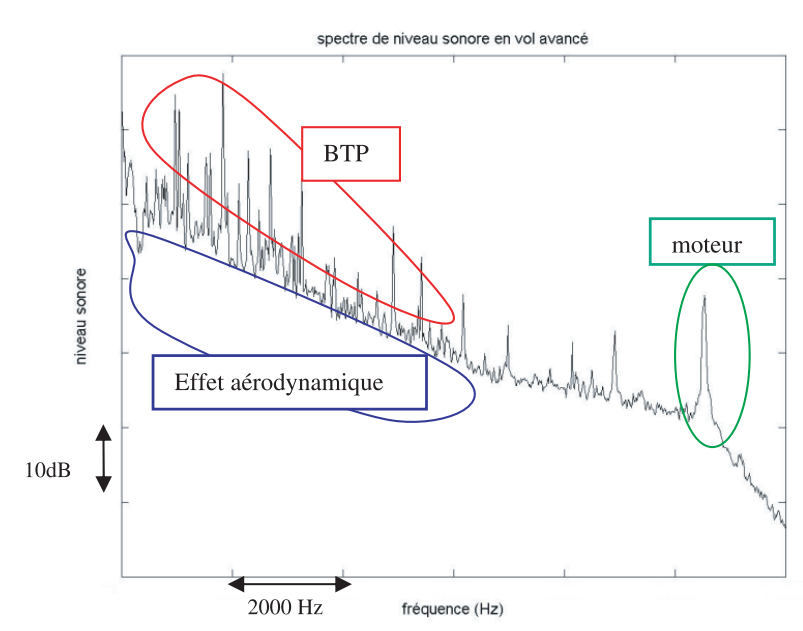

Fig. 2. Allure du spectre de niveau sonore interne.

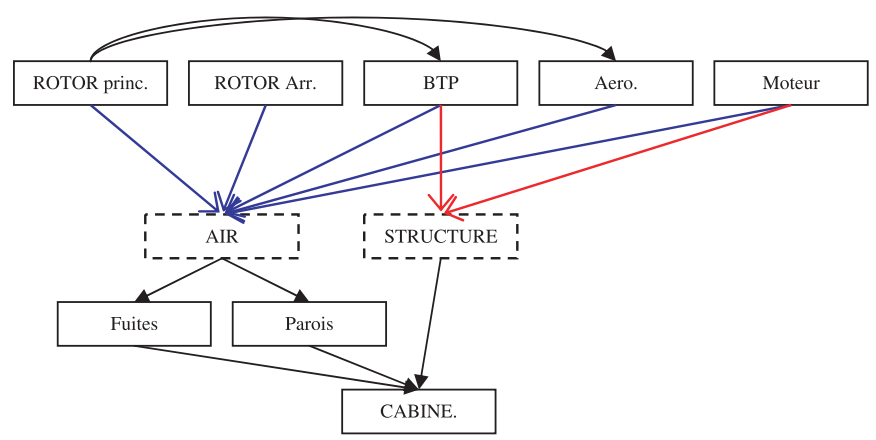

Fig. 3. Les chemins de bruit.

la structure est loin d'être négligeable (Fig. 3). La BTP est en effet fixée à la structure par l'intermédiaire de 4 barres au-dessus de la cabine. Cependant, les contributions aériennes et solidiennes sont mal identifiées.

Le bruit interne se présente donc comme un bruit large bande sur lequel vient se greffer un spectre de raies à fort niveau. Le champ interne est diffus étant donné qu'il est impossible de connaître la provenance des ondes en tout point de la cabine. 
Cette étude vise à identifier les contributions des différentes sources de bruit sur le niveau de bruit interne et à analyser les chemins de propagation (solidiens et aériens) de l'énergie depuis son origine jusqu'à la cabine à partir d'une modélisation de cette dernière. Cette étude s'inscrit dans une stratégie de type prédictif, dans le but d'intégrer le facteur acoustique interne dans la phase de conception des appareils. Elle a pour but de conduire à préconiser des solutions techniques pour améliorer le confort acoustique en cabine en agissant notamment sur les panneaux d'habillage. L'utilisation de certaines combinaisons de matériaux à des endroits judicieusement choisis peut apporter un gain considérable dans la lutte contre les vibrations et le rayonnement acoustique en réduisant la propagation des ondes sonores et vibratoires (par isolation, amortissement, absorption).

Notre volonté est, dans un premier temps, d'orienter cette étude à partir de données expérimentales réalisées en vol afin de prendre en compte tous les phénomènes associés au déplacement et à la déformation de la structure. Ces mesures de rayonnement acoustique sur la paroi interne de l'hélicoptère sont ensuite utilisées pour alimenter des modèles de simulation du comportement acoustique de la cabine et des chemins de bruit.

Les différentes méthodes de diagnostic de bruit interne et de localisation de sources acoustiques sont donc recensées dans l'optique de statuer sur le système le plus adapté à une structure complexe telle que l'hélicoptère. Cet article présente ensuite les outils de simulation utilisés pour exploiter les résultats obtenus. Enfin, un exemple simple basé sur des mesures en chambre sourde permet d'illustrer et de valider les performances de la méthode choisie.

\section{Détection, localisation, et analyse des sources de bruit}

L'ambiance acoustique à l'intérieur d'une cabine d'hélicoptère provient de la transmission par voies structurale et aérienne au travers des parois et de réflexions internes éventuelles. Vu de l'intérieur, il ne s'agit donc pas d'identifier des sources mais des zones de rayonnement sur la paroi interne (appelées sources par abus de langage), sans se préoccuper à ce stade de l'origine exacte du mécanisme. Du fait des effets de réflexion et de sources parasites présents dans la cabine, cette phase de diagnostic ne peut-être effectuée par simple mesure microphonique. La problématique ici est de caractériser finement une région source donnée complexe (sources multiples, étendues, présentant des cohérences spatiales...).

L'évolution des techniques intensimétriques et d'antennerie a apporté une nouvelle dimension à l'approche expérimentale en vibro-acoustique : à l'analyse du spectre fréquentiel vient s'ajouter celle de la structure spatiale de l'émission sonore. Cependant, ces méthodes nécessitent pour la plupart un nombre de capteurs et de voies d'acquisition de plus en plus élevé.

J.-C. Pascal ([1]) définit trois grandes classes de méthodes permettant de « capter $»$ un champ acoustique rayonné par une «source»: les méthodes optiques captant un champ vibratoire qui alimente ensuite un logiciel de calcul de rayonnement acoustique, les méthodes intensimétriques qui permettent de déterminer localement un vecteur intensité acoustique, et les méthodes utilisant des antennes de microphones parmi lesquelles on distingue essentiellement les techniques de formation de voies (« beamforming ») et la technique d'holographie acoustique de champ proche $\left(\mathrm{NAH}^{2}\right)$. La référence [2] présente un état de l'art sur les évolutions de ces techniques.

Dans le cas d'une cabine d'hélicoptère, plusieurs contraintes s'imposent au système de mesure et à la technique employée. Il s'agit de déterminer la solution la plus adaptée à un problème d'acoustique intérieur habitacle où le champ acoustique est diffus et où de nombreuses réflexions parasites peuvent venir troubler la mesure, tout en prenant en compte les problèmes associés à une mesure en vol, caractérisée par un coût important (le système choisi doit donc permettre de réaliser les mesures sur une durée limitée) et par des conditions de mesures difficiles (vibrations de l'appareil). Du fait de cette dernière remarque, les méthodes optiques telles que la vibrométrie et l'interférométrie laser s'avèrent inadaptées. Outre le diagnostic des fuites, la localisation et la hiérarchisation des zones de rayonnement prépondérantes dans la cabine, l'approche choisie doit également permettre de fournir les données à implanter en entrée d'un code de simulation à définir.

Trois approches sont envisagées ici : l'intensimétrie, la formation de voies, et l'holographie de champ proche.

\subsection{Intensimétrie}

L'intérêt industriel de cette méthode n'est plus à démontrer. Son principe et les erreurs engendrées sont largement détaillés dans [3]. Initialement prévue pour quantifier la puissance acoustique de machines, cette technique est également parfois utilisée pour localiser des sources.

Dans le cadre de notre étude, les avantages de la technique bien connue des deux microphones résident dans sa facilité d'utilisation car peu de matériel à embarquer est nécessaire pour une mesure, son faible coût, et la possibilité de mesurer la partie réactive du champ acoustique et les puissances acoustiques partielles.

Cependant, une mesure à l'aide d'une sonde intensimétrique ne donne accès aux valeurs de pression, vitesse et intensité que point par point. Ainsi, malgré l'utilisation de robots afin d'obtenir un maillage régulier d'une surface, cette méthode pouvant s'avérer utile pour une détection rapide de fuites acoustiques engendre une durée de mesure prohibitive dans le cadre du diagnostic complet d'une cabine d'hélicoptère.

2 Nearfield Acoustical Holography. 


\subsection{Formation de voies ( beamforming )}

Il s'agit, à partir d'une antenne composée de capteurs microphoniques, de calculer la pression sonore émise dans une direction particulière (différenciation des niveaux sonores basée sur la direction suivant laquelle ils parviennent à l'antenne). L'idée est d'augmenter le rapport signal sur bruit en combinant les mesures relatives à un grand nombre de capteurs et en appliquant une méthode de traitement du signal efficace. Le principe de cette technique est d'ajouter les signaux captés par les microphones de l'antenne avec des retards et des pondérations dépendant de la direction sur laquelle on veut se focaliser. Afin de créer une image acoustique (cartographie) de la surface source, il faut balayer toutes les directions possibles.

Pour une bande de fréquences donnée, les performances de l'antenne se mesurent en termes de pouvoir de résolution (capacité du réseau à séparer deux sources proches, ce que traduit la largeur du lobe principal) et de rapport signal sur bruit (capacité à identifier une source faible en présence d'une source prépondérante, traduite par le rapport entre le niveau des premiers lobes secondaires au lobe principal). Ces performances sont dictées par la taille de l'antenne et l'espacement et la disposition des microphones. Par conséquent, c'est le nombre de capteurs qui limitera les performances de l'antenne. Typiquement, le pouvoir de résolution est de l'ordre de la longueur d'onde, la taille du réseau et sa distance aux sources sont du même ordre de grandeur et supérieurs aux longueurs d'onde. Ainsi, dans [4], la forme de l'antenne est rendue optimale en disposant les microphones sur une spirale de façon à réduire l'importance des lobes de repliement spatial. Certains constructeurs utilisent des géométries d'antenne à pas variable, avec des microphones moins espacés au centre afin d'augmenter la gamme de fréquence d'analyse. D'autres utilisent des géométries circulaires ou en croix afin d'optimiser la répartition des microphones et de limiter leur nombre.

Au niveau de l'algorithmie, de nombreuses techniques sont développées pour adapter la théorie de la formation de voies à un problème intérieur habitacle. Dans [5], la théorie est adaptée en considérant des fronts d'ondes sphériques pour prendre en compte la faible distance entre l'antenne et le bruiteur.

Cependant, la méthode de formation de voies classique ne permet pas la quantification des puissances. Par contre, la technique appelée «focalisation de champ proche » dans [6] permet grâce à quelques innovations algorithmiques, d'adapter la méthode de formation de voies au champ proche et de pouvoir quantifier les puissances.

Avec les moyens d'acquisition actuels, cette technique présente l'avantage d'être très rapide (quelques secondes d'acquisition) puisque les traitements sont effectués numériquement après la mesure, et que le fait d'être suffisamment loin de la source permet de scanner de grandes surfaces. D'autre part, les résultats issus d'une mesure par formation de voies sont très faciles à interpréter, et il est également possible d'obtenir des cartographies 3D.
L'inconvénient majeur de cette technique est qu'elle nécessite l'hypothèse de champ libre, et il faut donc envisager de protéger l'antenne des autres parois et des réflexions parasites. C'est ce qui est fait dans [5] où l'expérimentation est effectuée avec de la mousse absorbante pour réduire l'effet des réflexions. En pratique, cela s'avèrerait trop compliqué de placer et déplacer en vol des panneaux de mousse dans une cabine d'hélicoptère. De plus, la grande envergure de l'antenne pose des problèmes pratiques de mesure dans le cas d'un habitacle.

Autre inconvénient important de cette technique, les résultats d'intensité et de vitesse ne sont pas accessibles. Or, dans le cadre de simulation, ces données peuvent s'avérer nécessaires et indispensables.

Les principales applications de la technique de formation de voies, du fait de l'hypothèse de champ libre, restent des applications de mesure de bruit externe. Ainsi, l'ONERA a utilisé des réseaux de microphones au cours de nombreux essais en soufflerie. Une autre des applications importantes de la formation de voies est la mesure de bruits non-stationnaires, et en particulier pour l'étude du bruit de passage des véhicules (Citroën, SNCF), comme dans [7]. D'autres applications sont présentées dans [8] et $[6]$.

\subsection{Holographie acoustique de champ proche}

L'holographie acoustique de champ proche, ou Nearfield Acoustical Holography $(\mathrm{NAH})$, est désormais largement appliquée à la localisation de sources. L'objet de cette méthode est de résoudre le problème inverse, à savoir, à partir de données mesurées sur un réseau $2 \mathrm{D}$ de microphones, de reconstituer le champ sur la surface de la cabine (plan source, situé à une distance $d$ de l'antenne). Son principe est cité dans de nombreuses références (voir $[9,10]$ ). Il peut être décomposé en trois étapes (notations explicitées dans la nomenclature) :

- Le rayonnement acoustique capté par l'antenne est décomposé en composantes planes par transformée de Fourier spatiale :

$$
\begin{aligned}
& P_{\mathrm{A}}\left(k_{x}, k_{y}, z=z_{\mathrm{A}}, \omega\right)= \\
& \frac{1}{(2 \pi)^{2}} \iint_{x, y} p_{\mathrm{A}}\left(x, y, z=z_{\mathrm{A}}, \omega\right) \mathrm{e}^{j\left(k_{x} x+k_{y} y\right)} \mathrm{d} x \mathrm{~d} y= \\
& \operatorname{SFT}\left(p_{\mathrm{A}}(x, y, z, \omega)\right)
\end{aligned}
$$

- Un opérateur de propagation inverse sous la forme d'une fonction de Green $(G)$ est ensuite appliqué afin de rétropropager les composantes sur le plan source :

$$
P_{\mathrm{S}}\left(k_{x}, k_{y}, z=z_{\mathrm{S}}, \omega\right)=P_{\mathrm{A}}\left(k_{x}, k_{y}, z=z_{\mathrm{A}}, \omega\right) G(K, d)
$$


$G$ prend deux formes différentes suivant la nature des ondes traitées, évanescentes ou propagatives :

$$
G(K, d)=\left\{\begin{array}{r}
\mathrm{e}^{j \sqrt{k^{2}-k_{x}^{2}-k_{y}^{2}} d} \text { pour les ondes } \\
\text { propagatives, } k_{x}^{2}+k_{y}^{2} \leq k^{2} \\
\mathrm{e}^{j \sqrt{k_{x}^{2}+k_{y}^{2}-k^{2}} d} \text { pour les ondes } \\
\text { évanescentes, } k_{x}^{2}+k_{y}^{2}>k^{2}
\end{array}\right.
$$

- Le champ de pression sur le plan source est finalement reconstitué par l'intermédiaire d'une transformée de Fourier spatiale inverse.

$$
p_{\mathrm{S}}(x, y, z, \omega)=S F T^{-1}\left\{P_{\mathrm{S}}\left(k_{x}, k_{y}, z, \omega\right)\right\}
$$

Cependant, cette technique comporte tout de même quelques limitations, et réclame des algorithmes de traitement particuliers. D'une part, des distorsions sont introduites par la troncature spatiale du champ acoustique à cause de la nature finie de l'antenne. Ce phénomène est atténué par une opération de filtrage du spectre de nombre d'ondes. La référence [11] présente également une nouvelle technique réduisant les erreurs dues à l'utilisation de la transformée de Fourier spatiale en effectuant la reconstruction holographique non plus dans le domaine des nombres d'ondes mais dans le domaine spatial. D'autre part, de nombreux algorithmes de traitement sont appliqués pour réduire les distorsions engendrées par l'amplification exponentielle des ondes évanescentes bruitées. Parmi les techniques appliquées, on citera notamment le filtrage de Wiener [12], la régularisation de Tikhonov, et les méthodes de décomposition en valeurs singulières. Pour plus d'informations sur ce sujet, voir [13].

Dans le cas de la cabine d'hélicoptère, les phénomènes de génération de bruit seront considérés stationnaires, et les techniques de l'holographie plane seront utilisées (courbure des faces latérales négligées). L'étude de phénomènes stationnaires permet de ne pas mesurer tous les points du maillage simultanément, et un nombre réduit de microphones peut être déplacé pour couvrir la source, à condition de conserver une référence de phase. Ce traitement est illustré dans [1].

Les principaux avantages de l'holographie acoustique de champ proche résident dans le fait que la mesure est effectuée en champ proche, ce qui permet d'une part d'augmenter le rapport signal à bruit et d'autre part de prendre en compte les ondes évanescentes, ce qui conduit à une meilleure résolution.

D'autre part, les données de pression, de vitesse, d'intensité, de puissance, de cohérence avec une référence fixe, ainsi que les diagrammes de directivité des sources sont accessibles par cette technique facilitant ainsi l'emploi ultérieur d'outils de simulation.

Une des principales limitations de cette technique est sa durée d'utilisation dans le cas d'un habitacle de véhicule : préparation des essais (maillage de la cabine) et durée importante des mesures elles-mêmes. Un autre inconvénient est la quantité importante de matériel à embarquer.
Les applications industrielles du principe d'holographie sont très diverses. Dans [14], l'holographie acoustique de champ proche est appliquée à l'habitacle d'un avion Beech 1900D en vol. Il y a également de nombreuses applications de l'holographie pour la mesure du bruit de moteurs, et également de nombreuses applications de l'holographie à l'étude de bruits de passages [15].

Il est à noter que l'holographie acoustique de champ proche peut également être couplée avec une technique d'analyse inverse appelée IBEM (voir [16]) pour reconstruire le champ vibratoire sur des éléments mécaniques de forme quelconque à partir de mesures de pression sur un réseau de microphone.

\subsection{Choix effectué, configuration}

Après analyse des avantages et inconvénients des différentes méthodes, notre choix se porte sur l'holographie acoustique de champ proche. Il semble en effet que ce soit la méthode la plus appropriée pour une mesure complète d'un habitacle de véhicule particulièrement bruité. D'autre part, la quantité de données accessibles (pression, vitesse, intensité), et le fait que ces données soient quantifiées, laisse envisager plus de possibilités en termes de couplage avec un outil de simulation. Il faut cependant analyser ses résultats en milieu confiné et particulièrement bruité afin de valider son application à une cabine d'hélicoptère. Des résultats convaincants sont présentés dans [17].

La taille de l'antenne et l'espacement entre les microphones définissant la gamme de fréquences analysable $\left(f_{\max }=c / 2 d d\right.$ étant la distance entre deux capteurs) et la résolution obtenue, le choix du nombre et de la disposition des microphones est très important. Le matériel retenu est une antenne carrée de 64 micros $(8 \times 8$ micros $)$ espacés de $2,5 \mathrm{~cm}$, et un système d'acquisition comportant au moins 68 voies d'acquisition, du fait de la nécessaire utilisation de quatre voies de références. Les quatre capteurs de références (microphones ou accéléromètres) seront placés judicieusement de manière à distinguer l'influence de la BTP, du bruit aérodynamique et des autres sources de bruit.

Avec un tel système, la durée de mesure nécessaire pour analyser la surface de la cabine est estimée à environ deux heures, ce qui reste admissible dans notre application, et cela pour une bande de fréquence allant jusqu'à $6800 \mathrm{~Hz}$.

La mise en pratique d'une mesure par holographie acoustique est détaillée dans [18].

\section{Modélisation et simulation du comportement vibro-acoustique}

Les difficultés associées à la simulation du comportement vibro-acoustique de la cabine sont liées à la largeur de bande de fréquence analysée, impliquant l'utilisation de méthodes différentes pour les domaines basses fréquences et moyennes/hautes fréquences. 


\subsection{Outils basses-fréquences}

Les méthodes numériques classiques $\mathrm{FEM}^{3}$ et $\mathrm{BEM}^{4}$ ([13]), qui reposent respectivement sur des modèles éléments-finis de volume et de surface, sont bien adaptées aux basses fréquences, y compris pour des structures complexes. On trouve de nombreuses applications de ces méthodes dans la littérature, et notamment à des habitacles de véhicule $[19,20]$. Ces méthodes transforment l'équation de Helmoltz et les conditions aux limites associées en un problème algébrique résolu numériquement.

Cependant, pour obtenir un niveau de précision acceptable lors de la détermination des pressions acoustiques et des déplacements structuraux, la règle est généralement d'utiliser 10 éléments par longueur d'onde acoustique. Par conséquent, la complexité des modèles éléments finis augmente exponentiellement avec la fréquence et ces méthodes deviennent inadaptées aux moyennes et hautes fréquences.

\subsection{Outils hautes-fréquences}

Aux moyennes et hautes fréquences, il existe principalement deux catégories de méthodes de prévision acoustique : la SEA et les méthodes géométriques. La SEA ${ }^{5}$ est une approche basée sur des considérations statistiques et énergétiques. Les possibilités actuelles de cette méthode restent inappropriées pour un système complexe tel que l'hélicoptère.

Une méthode géométrique basée sur le tir de rayons est donc utilisée pour caractériser le comportement acoustique de la cabine en hautes fréquences $(400-6000 \mathrm{~Hz})$ par l'intermédiaire du logiciel ICARE, fourni par le CSTB. À ces fréquences, comme cela est suggéré dans [21], le couplage vibro-acoustique est beaucoup plus faible, et il est par conséquent envisageable d'ignorer l'effet de la pression acoustique dans la cabine sur la vibration de la structure. Cette hypothèse effectuée par P. Jean [22], permet de combiner l'approche intégrale et l'approche géométrique et ainsi de résoudre le problème d'une source surfacique rayonnante (cas d'un panneau vibrant). En effet, la formulation intégrale bien connue du problème acoustique est :

$$
\begin{aligned}
& P(M, \omega)= \\
& \int_{S_{\mathrm{f}}}\left[G(Q, M, \omega) \frac{\partial P(Q, \omega)}{\partial n}-P(Q, \omega) \frac{\partial G(Q, M, \omega)}{\partial n}\right] \mathrm{d} S_{\mathrm{f}}(Q)
\end{aligned}
$$

où $M$ est le point fluide où la pression acoustique $P$ est évaluée (typiquement les oreilles des passagers), $S_{\mathrm{f}}$ est la surface frontière de la cavité et $G$ est une fonction de Green solution du problème. L'idée de la

\footnotetext{
${ }^{3}$ Finite Element Method.

4 Boundary Element Method.

5 Statistical Energy Analysis.
}

méthode dénommée GRIM $^{6}$ formulée par P. Jean est de calculer la fonction de Green $G_{\mathrm{V}}$ incluant toutes les réflexions sur les parois de la cabine avec un algorithme géométrique (ICARE), puis d'ignorer l'effet du couplage vibro-acoustique en supposant la surface $S_{\mathrm{V}}$ rigide

$$
\begin{aligned}
& \left.\frac{\partial G_{\mathrm{V}}(Q, M, \omega)}{\partial n}\right|_{S_{\mathrm{V}}}=0 \\
& \left.\frac{\partial P(Q, \omega)}{\partial n}\right|_{S_{\mathrm{V}}}=j \omega \rho \vec{V}(Q, \omega) \cdot \vec{n} \quad \text { (Euler) }
\end{aligned}
$$

En utilisant ces hypothèses, il est possible de relier la pression $P$ en tout point $M$ intérieur à la cabine due à la vibration de la surface $S_{\mathrm{V}}$ par l'équation :

$$
P(M)=\int_{S_{\mathrm{V}}} j \omega \rho V(Q) G_{\mathrm{V}}(M, Q) \mathrm{d} S(Q)
$$

Le calcul de la fonction de Green $G_{\mathrm{V}}$ est effectué par tir de rayons. La contribution acoustique de chacun des trajets calculés nécessite les impédances acoustiques des matériaux présents dans la cabine; celles-ci sont déterminées à partir de mesures au tube de Kundt. D'autre part, un des paramètres d'entrée fondamentaux de cette approche GRIM est la vitesse acoustique sur la surface du panneau vibrant étudié. Celle-ci peut être introduite par l'intermédiaire des résultats de vitesses obtenus par holographie de champ proche, où par un calcul BEM.

\subsection{Couplage avec le système de mesure}

Afin d'utiliser le logiciel ICARE, une modélisation de la cabine d'un hélicoptère a été effectuée à partir d'un modèle de l'habillage intérieur de cette dernière (Fig. 4).

À partir de cette modélisation géométrique et des résultats des mesures effectuées avec l'antenne, il est possible d'analyser le comportement vibro-acoustique hautes-fréquences d'une cabine d'hélicoptère selon la méthode suivante (illustrée dans le Tab. 1), et largement détaillée dans [23] :

a) dans un premier temps, des mesures en vol sont effectuées avec l'antenne de mesure. Le traitement holographique effectué en post-traitement permet de reconstruire des cartographies 2D de pression, vitesse et intensité acoustique dans le plan des sources (les surfaces internes de la cabine d'hélicoptère). Ces cartographies permettent la détection efficace des fuites acoustiques et la localisation des panneaux rayonnant de façon prépondérante.

b) Dans un deuxième temps, le calcul des fonctions de Green $\left(G_{\mathrm{V}}\right)$ associées à chacun des panneaux sources mesurés est effectué (calcul par tir de rayons). Ce dernier permet de localiser les zones de la cabine soumises à de nombreuses réflexions (b).

${ }^{6}$ Green Ray Integral Method. 
Tableau 1. Principe de la méthode.

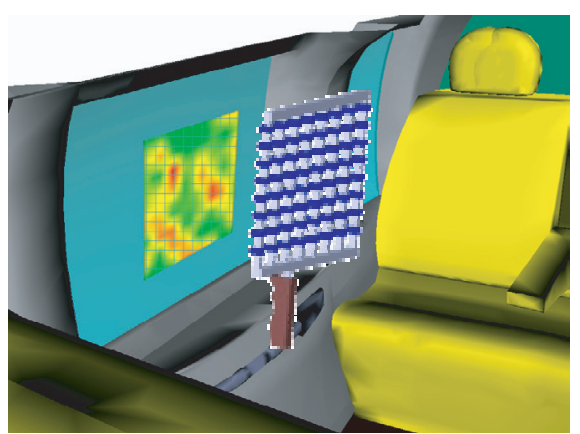

( a )

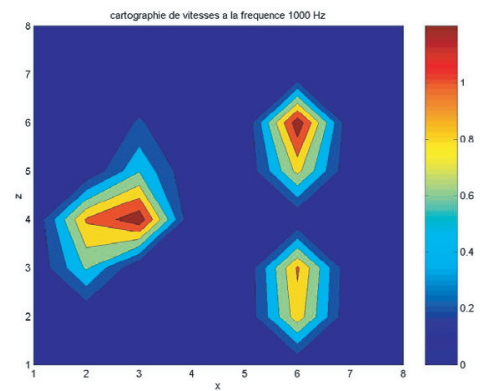

(c)

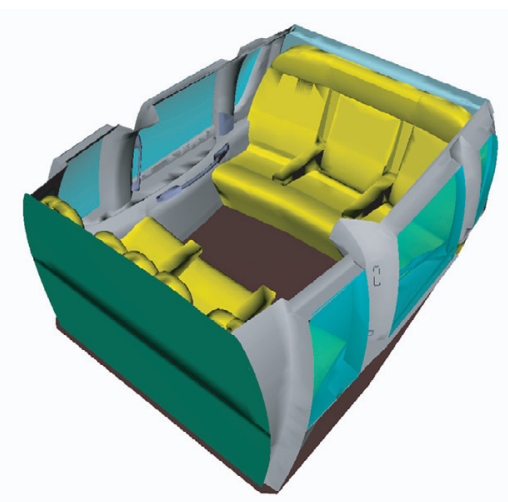

Fig. 4. Modélisation de la cabine d'un hélicoptère, en configuration VIP.

c) Ensuite, les résultats de vitesses obtenus en (a) sur les surfaces de la cabine par holographie sont convertis en fichiers permettant d'alimenter le code de calcul Icare $(V(Q)$ dans l'équation $(7))$.

d) Les propriétés d'absorption acoustiques des matériaux de la cabine mesurées au tube de Kundt sont introduites dans le modèle de simulation. Les contributions des différentes surfaces de la cabine en termes de pression acoustique aux oreilles des passagers sont déduites des étapes précédentes.

Enfin, et dans un but de validation du modèle, les contributions de toutes les sources mesurées sont sommées et comparées à des spectres de pression mesurés aux oreilles des passagers. Le modèle est ensuite utilisé pour

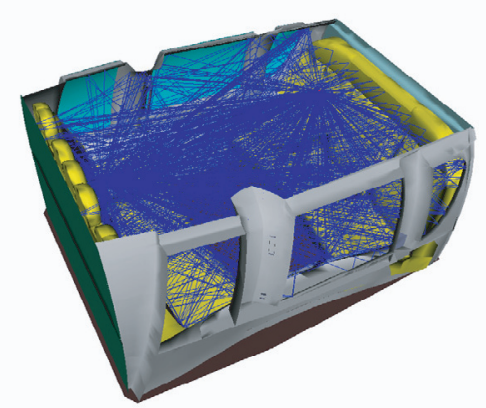

(b)

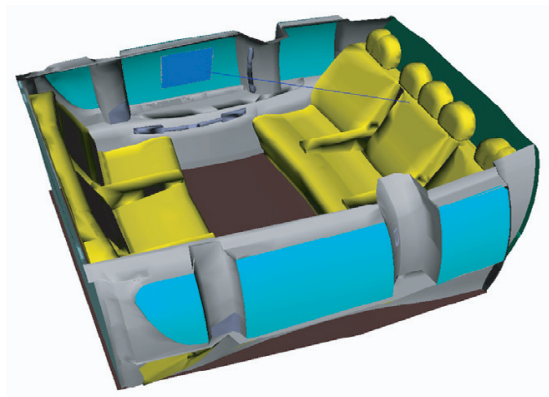

(d)

quantifier l'influence d'ajout de matériaux absorbants notamment.

\subsection{Exemple de résultats}

La méthode présentée ici a été appliquée et validée dans le cas d'un hélicoptère. Des mesures effectuées par holographie acoustique sur la cloison arrière excitée artificiellement par des haut-parleurs placés dans la soute à bagage et générant un bruit blanc ont été introduites dans le modèle d'acoustique géométrique de la cabine. Les pressions acoustiques calculées et mesurées aux oreilles du copilote sont comparées sur la figure 5 . Les résultats obtenus montrent le très bon comportement de la méthode dans un environnement confiné et complexe, pour toute la gamme de fréquence du dB SIL4. En outre, et comme prévu étant donné les hypothèses de l'acoustique géométrique et les limitations de l'holographie acoustique, la méthode reste limitée en bassesfréquences (résultats non présentés). Néanmoins, les outils décrits à la section 2.1 sont plus adaptés dans ce cas, et la méthode choisie s'avère pleinement satisfaisante.

\section{Conclusion}

Le problème était de définir un système allant de la mesure jusqu'à l'exploitation des résultats par la modélisation du comportement vibro-acoustique d'une cabine d'hélicoptère. Il a donc fallu analyser les différents 


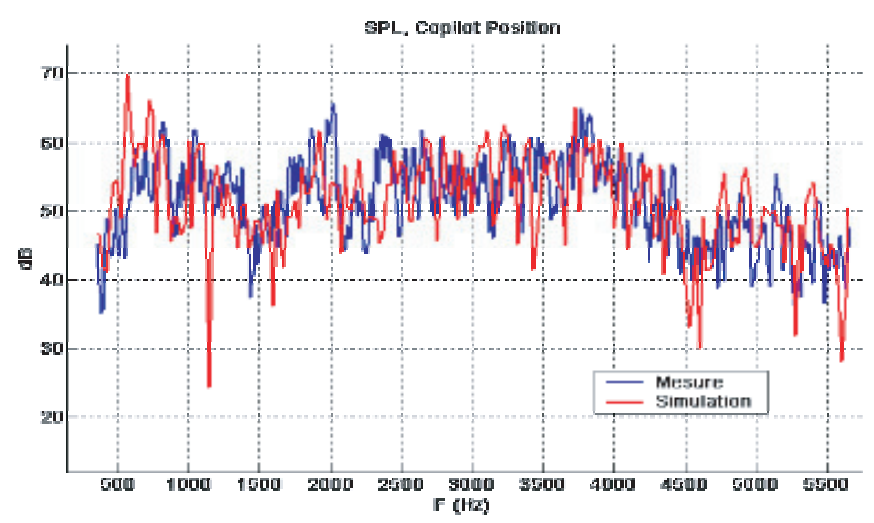

Fig. 5. Résultats de la méthode sur hélicoptère.

choix possibles et les méthodes sous-jacentes, et faire un choix pertinent adapté au cas de la structure complexe qu'est l'hélicoptère. Les différentes techniques d'imagerie acoustique ont été recensées afin d'en analyser les avantages et les inconvénients. Les techniques d'intensimétrie, de formation de voies, et d'holographie acoustique de champ proche possèdent chacune des avantages et pourraient s'avérer complémentaires. Le choix s'est porté sur l'holographie acoustique, du fait de l'avantage d'une mesure en champ proche et de la quantité des données accessibles en post-traitement. En effet, l'objectif de cette étude est aussi la modélisation acoustique. Par conséquent, les différents outils de simulation ont également été répertoriés. La gamme de fréquence analysée et les possibilités de prendre en compte le rayonnement de panneaux mesuré par holographie font de ICARE un outil complémentaire à la mesure et adapté à notre problème.

Finalement, le système choisi couple mesures expérimentales basées sur l'holographie acoustique de champ proche et simulation acoustique à partir d'une méthode géométrique. Cette approche devrait permettre d'analyser la contribution des surfaces rayonnantes de la cabine, et également de prévoir l'influence de changements de matériaux (ajout de matériaux absorbants notamment).

Les épreuves n'ont pas été relues par les auteurs.

\section{Références}

[1] J.-C. Pascal, Les techniques d'imagerie acoustique et l'holographie acoustique, Journée imagerie acoustique appliquée, CRITT-M2A, 5 novembre 2002

[2] M.B.S. Magalhaes, R.A. Tenenbaum, Sound Sources Reconstruction Techniques: a review of their Evolution and New Trends, Acta Acoustica 90 (2004) 199-220

[3] C. Lesueur, Rayonnement acoustique des structures, Eyrolles, 1988

[4] J. Hald, J.J. Christensen, A class of optimal broadband phased array geometries designed for easy construction, Proceedings of Inter-Noise 2002, Dearborn, MI, USA, 19 21 août 2002

[5] K. Haddad, V. Benoit, Capabilities of a beamforming technique for acoustic measurements inside a moving car,
Proceedings of Inter-noise 2002, Dearborn, MI, USA, 1921 août 2002

[6] B. Béguet, Les techniques de localisation et mesures d'isolation acoustique, Confort automobile et ferroviaire, 1314 novembre 2002

[7] M.-A. Pallas, The focused vertical array for the description of the noise sources on a moving car, Proceedings of Inter-noise 2000, Nice, France, 27-30 août 2000

[8] R. Boscher, J. Chatillon, Wide band acoustic imaging of moving sources using synthetic aperture sonar, Application to transportation Noise, Proceedings of Inter-noise, Nice, 27-30 août 2000

[9] J.D. Maynard, E.G. Williams, Y. Lee, Nearfield Acoustical Holography: 1. Theory of generalized Holography and the development of NAH, 1985, J.A.S.A., pp. 1395-1413

[10] J. Hald, STSF - a unique technique for scan-based Nearfield Acoustic Holography without restrictions on coherence, B\&K Technical Review 1 (1989) 1-50

[11] R. Steiner, J. Hald, Near-field acoustical holography without the errors and limitations caused by the use of spatial dft, IJAV, June 2001

[12] D. Vaucher de la Croix, P. Chevret, F. Perrin, Use of Acoustical Holography in 3D Interior measurements, Proceedings of Inter-noise 2002, Dearborn, MI, USA, 1921 August 2002

[13] E. Williams, Regularization and Nearfield Acoustical Holography, Proceedings of Inter-Noise 2001, The Hague, The Netherlands, 27-30 August 2001

[14] E.G. Williams, B.H. Houston, P.C. Herdic, R. Raveendra, B. Gardner, Interior near-field acoustic holography In flight, J.A.S.A 108 (2000) 1451-1463

[15] J. Hald, Use of Spatial Transformation of Sound Fields (STSF) Techniques in the Automotive Industry, Brüel \& Kjaer Technical Review 1 (1995)

[16] A.P Schumacher, P.C. Hansen, Sound Source Reconstruction Using Inverse BEM, Proceedings of Inter-Noise 2001, The Hague, The Netherlands, 27-30 August 2001

[17] J. Caillet et al., Nearfield Acoustical Holography Measurement Inside a helicopter cabin, Proceedings of European Test \& Telemetry Conference (ETTC05), Toulouse, France, 7-9 June 2005

[18] S.M Dumbacher, D.L Brown, Practical Aspects of making NAH measurements, Society of Automotive Engineers, 1998

[19] A.R. Mohanty, B.D. St Pierre, P. Suruli-Narayanasami, Structure-borne noise reduction in a truck cab interior using numerical techniques, Applied Acoustics 59 (2000) $1-17$

[20] W. Desmet, B. Pluymers, P. Sas, Vibro-Acoustic Analysis procedures for the evaluation of the sound insulation characteristics of agricultural machinery cabins, J.S.V. 266 (2003) 407-441

[21] C. Floc'h, A. Bardot, X. Bohineust, J.-D. Polack, Vibroacoustic simulation using geometrical acoustics in the medium frequency range inside a car cavity, Proceedings of Inter-Noise 2000, Nice, France, 27-30 August 2000

[22] P. Jean, Coupling integral and geometrical representations for vibro-acoustical problems, J.S.V. 224 (1999) 475-487

[23] J. Caillet et al., Diagnosis and modelling of interior noise in helicopter cabins, European Rotorcraft Forum 2005, Florence, Italie, 13-15 September 2005 